\title{
Saúde da Família, agora, mais do que nunca?
}

\author{
Claunara Schilling Mendonça
}

RESUMO

Motivação: Sintetizar os processos avaliativos realizados a partir do Ministério da Saúde ao longo da expansão da Saúde da Família e dos compromissos assumidos pelo Brasil como parte dos esforços globais para garantia do acesso universal aos serviços de saúde

\section{Método: Artigo de opinião}

Resultados: Os resultados indicam que o aumento do acesso da população brasileira à ESF, um modelo de atenção primária à saúde que leva em conta os determinantes sociais e estruturais da saúde, foram decisivos na ampliação do acesso, na equidade e impactou nos clássicos indicadores de saúde materno-infantil e na redução das mortes precoces por doenças crônicas não transmissíveis.

Conclusões: 0 alcance de uma infraestrutura mínima, de insumos e de profissionais, leva nosso país à um novo patamar de protagonismo global que é colocar a APS/Saúde da Família como centro das redes de atenção em todo o país. Dar visibilidade ao já conquistado é uma das formas de garantir a sustentabilidade da ESF e do SUS, em um contexto restritivo de políticas sociais e de austeridade econômica, que enfrentamos nesse início do século 21.

Palavras-chave: Atenção Primária à Saúde; Sistema Único de Saúde; Saúde da Família; acesso aos serviços de saúde
Revista da Rede APS 2019

Publicada em: 17/12/2019

DOI:10.14295/aps.v1i3.48

Claunara Schilling Mendonça (Pesquisadora do Centro de Estudo e Pesquisa em APS/ GHC. Docente do Mestrado Profissional em Avaliação e Produção de Tecnologias para o SUS. Professora do Departamento de Medicina Social da Faculdade de Medicina da Universidade Federal do Rio Grande do Sul);

\section{Correspondência para:} claunara@ghc.com.br; cmendonca@hcpa.edu.br 


\section{INTRODUÇÃO}

As considerações aqui apresentadas visam descrever os avanços alcançados pela Estratégia Saúde da Família, e os desafios que enfrenta, ainda hoje, em assumir seu papel como porta de entrada e coordenadora da atenção no sistema de saúde.

A cultura da avaliação de políticas públicas é ainda incipiente no Brasil e num contexto de escassez de recursos e políticas de austeridade fiscal, torna-se imprescindivel conhecer os resultados obtidos pelos programas/políticas para a tomada de decisão que maximize o impacto e os resultados na sociedade. Em que pese o Programa de Saúde da Família não ter tido um planejamento inicial de realizar avaliação de impacto, quantificando relações causais entre o programa e um conjunto de resultados na sociedade, desde sua criação, processos avaliativos foram implantados.

De 1998, ano da primeira grande inflexão para o crescimento da Saúde da Família, que foi o pagamento do Piso de Atenção Básica, permitindo, pela primeira vez no país, um repasse financeiro do Ministério da Saúde a todos os municípios brasileiros, de acordo com sua população, até o ano de 2006, quando foi publicada a Política Nacional de Atenção Básica, tornando o que até então era um programa, numa Política Nacional, a cobertura populacional evoluiu de 5 a 45,3\%, alcançando 84 milhões de brasileiros em 2006 até os mais de 100 milhões de brasileiros (64\%) em setembro de 2019, com 43.508 equipes implantadas.

A expansão da Saúde da Família no Brasil se deu de forma heterogênea no país, crescendo inicialmente em municípios menores, com piores Índices de Desenvolvimento Humano e infraestruturas inadequadas.

Na primeira avaliação normativa realizada no país, pelo Ministério da Saúde, em 2001/2002, existiam 13.501 equipes implantadas em 3.778 (68\%) municípios em todo território nacional. A sustentabilidade do então programa era questionada, tanto pelo seu subfinanciamento, como pela dificuldade na contratação de médicos pelos gestores municipais, estruturas precárias, falta de insumos, principalmente medicamentos, que poderia resultar em uma assistência à saúde de baixa qualidade, uma atenção primária seletiva, focalizada, semelhante a outras concepções de APS na América Latina. Nesse contexto, um censo de todas as equipes existentes é realizado, e achados de uma implantação incipiente do programa no Brasil são encontrados, como $77 \%$ dos médicos há menos de um ano nas equipes, $81,4 \%$ das Unidades Básicas com a presença de todos os equipamentos considerados básicos naquele momento (tensiômetro, estetoscópio, termômetro, balança infantil e adulta), com enormes variações regionais. Em 3\% das unidades não havia pelo menos UM consultório médico e em $38 \%$ delas não havia consultório de enfermagem. Embora 93\% das equipes odontológicas dispunham de cadeira odontológica, $26 \%$ dessas não tinham o instrumental completo. A presença (e não suficiência) dos medicamentos também era muito deficitária, com 30\% das UBS sem acesso a antihipertensivos e $60 \%$ sem acesso a antibióticos. Somente $55 \%$ das equipes com acesso a exames complementares básicos e $47,3 \%$ com disponibilidade de internação hospitalar

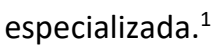

A situação dos clássicos indicadores de saúde que indicam a evolução do acesso aos sistemas de saúde, como as taxas de mortalidade, eram, em 2001, de 27,5/1.000 Nascidos Vivos (NV) para a mortalidade infantil; de cada 100 mil NV, 73,3 mulheres morriam e de cada 100 mil óbitos, 500 eram óbitos prematuros por doenças crônicas não transmissíveis.

E o contexto internacional exigia ações do Brasil para o alcance das Metas dos Objetivos do Milênio (ODMs), com oito objetivos internacionais de desenvolvimento estabelecidos após a Cúpula do Milênio das Nações Unidas em 2000, que propunha para o Objetivo 4 a redução da Mortalidade Infantil para menos de 15,7 por 1000 nascidos vivos no ano de 2015. O aumento do acesso da população aos serviços de saúde e as políticas de distribuição de renda como o Bolsa Família, fez com que o Brasil alcançasse, já em 
2010, a meta pactuada de redução da Mortalidade Infantil. Nos anos subsequentes, o monitoramento das metas dos ODM resultou no compromisso, entre o Ministério da Saúde e os Estados e Municípios, do Pacto pela Redução da Mortalidade Infantil nas Regiões da Amazônia Legal e Nordeste do Brasil (PRMI), cujo objetivo principal era o de reduzir em 5\% ao ano a TMI nos anos de 2009 e 2010, em 154 municípios do Nordeste e 102 municípios da Amazônia Legal ${ }^{2}$. Essa meta, que para o componente pós-neonatal, propunha a implantação de ESF nesses municípios, foi baseada em estudo publicado em 2006 por Macinko, Guanais e Souza, que mostrava associação entre o aumento de $10 \%$ de cobertura da SF e a redução de 4,5\% na mortalidade infantil, controladas outras variáveis associadas à $\mathrm{Ml}^{3}$

Em 2002 mais de 29 mil equipes estavam implantadas, representando $50 \%$ de cobertura populacional brasileira. No entanto, a cobertura nos municípios com mais de 100 mil habitantes era de $22 \%$. Uma iniciativa do MS, apoiada pelo Banco Mundial e BIRD, criou o Projeto de Expansão e Consolidação da Saúde da Família - PROESF, a fim de mudar essa realidade nos grandes municípios, com investimentos de 550 milhões de dólares, em 8 anos, com um dos seus componentes sendo de Monitoramento e Avaliação ${ }^{4}$. Foram realizados Estudos de Apoio à expansão da SF, conhecidos como Estudos de Linha de Base, nos então 231 municípios brasileiros ${ }^{5}$. Os resultados apresentados pelas diferentes instituições acadêmicas envolvidas nessas pesquisas, por inconsistências com as dimensões analíticas propostas no Termo de Referência, que eram implantação e impacto, organização $e$ integralidade do cuidado, desempenho dos sistemas locais e o contexto político, não criaram, de fato, uma linha de base e, por diferentes critérios avaliativos e rigor metodológico, análises de inferências causais desse programa não foram conclusivas 6,7 . A cobertura média de SF desses municípios, no entanto, saiu de 24 para $40 \%$ no período do Projeto, e continuaram sua expansão continuamente.

É do ano de 2008 um novo estudo amostral, financiado pelo Ministério da Saúde, a fim de avaliar a estrutura da ESF no Brasil e permitir comparar com o momento anterior (2001/2002) ${ }^{8}$. Em comparação com o censo de 2001, 55\% das equipes estavam em funcionamento há mais de 5 anos (4\% em 2001), embora 7\% do total das ESF visitadas estivessem sem médico. Houve eliminação do percentual de unidades sem nenhum consultório médico por equipe e redução de $20 \%$ das unidades de saúde sem nenhum consultório de enfermagem por equipe. Demais instalações como sala de vacinas nas unidades de saúde aumentaram $10 \%$ entre as duas avaliações e a presença dos equipamentos de informática, presentes em somente $25 \%$ das Unidades Básicas, ainda assim representaram um aumento de $11 \%$ em relação a 2001/02. Ao avaliar a estrutura física mínima para funcionamento das UBS, $74,65 \%$ das 1.857 UBS avaliadas estavam abaixo do padrão mínimo de instalações, concentrados na baixa presença de sala específica para dispensar medicamentos e sala de expurgo. Esses resultados foram utilizados para a criação de uma portaria que financiaria, pela primeira vez no país, investimentos no formato fundo a fundo, para obras de construção de Unidades Básicas de Saúde e barcos na Amazônia legal, a fim de reduzir o déficit estrutural desses serviços. Previam a construção de 8.694 UBS em 4 anos, com um impacto financeiro de 1,738 bilhões ${ }^{9}$. Esses recursos, ampliados posteriormente pelo Programa "Requalifica UBS", que incluiu financiamento para reformas e ampliações de UBS, financiaram 16.331 obras em UBS em todo o país 10.

Nos anos de 2008 e 2009, foi realizado um inquérito epidemiológico transversal de base populacional, a fim de avaliar o desempenho da rede de serviços de saúde, numa amostra da população urbana composta por crianças até os cinco anos de idade (6.307), adultos de 20 até 59 anos (12.402), e idosos com 60 anos ou mais de idade (6.624), residentes em municípios de pequeno, médio e grande porte, representativa das macroregiões do país ${ }^{11}$. Esse estudo foi chamado de "White" brasileiro, fazendo referência ao clássico estudo de 1961, The ecology of medical care $^{12}$ repetido por Green para a população estadunidense, em $2001{ }^{13}$. Dos indivíduos entrevistados, $34,5 \%$ consultaram um médico nos últimos três meses, enquanto na população dos EUA foi de 21,7\%, 77,6\% em Unidades Básicas de Saúde, nos EUA (11,3\%). Enquanto $24,4 \%$ dos brasileiros consultaram com especialistas $(4,1 \%$ no privado), $19 \%$ dos estadunidenses consultaram 
com especialistas. A assistência domiciliar foi recebida por $1,4 \%$ nos EUA e no Brasil, por 2,9\%, sendo que $11,7 \%$ dos idosos receberam atendimento domiciliar nos últimos três meses. Enquanto que 1,3 \% dos norteamericanos foram atendidos em urgências/emergências, no Brasil, foram $4,3 \%$, concentrados nas crianças $(10,4 \%)$. Em relação às hospitalizações, $0,8 \%$ da população do estudo norte-americano internou, e no Brasil, 1,3\% dos amostrados tiveram uma internação hospitalar.

A referência da SF como serviço de escolha da população foi verificada em outros estudos de base populacional como a Pesquisa Nacional de Saúde (PNS), cuja proporção de pessoas cadastradas na SF era $56,2 \%$, maior cobertura rural (71\%) que urbana $(50,6 \%)$, com baixa renda, $54 \%$ dos domicílios com rendimento mensal de até 2 salários mínimos e $66,3 \%$ dos chefes do domicílio com menos de um ano de escolaridade ${ }^{14}$. Essa realidade, se por um lado reforça a dimensão da equidade da SF, traz a necessidade de incorporar outros resultados - do cuidado recebido - na saúde da população, implementando metodologias capazes de avaliar o cumprimento da funcionalidade dos serviços de atenção primária: acesso e utilização integrais, ao longo do tempo e com coordenação na rede de atenção à saúde.

Em 2011, com motivação de ampliar o investimento na atenção primária, o Ministério da Saúde implanta o Programa Nacional de Melhoria de Acesso e da Qualidade da Atenção Básica $P M A Q$, que faz uma avaliação de estrutura e processo a partir de entrevistas a profissionais, gestores municipais e usuários, além de avaliação externa feita por Instituições Acadêmicas de Ensino Superior, e a provisão de incentivos financeiros foram alocados a partir da performance dessas entrevistas ${ }^{15}$. Esse Programa (PMAQ) produziu muitas publicações, que dizem respeito à estrutura das unidades, processos de trabalho abrangência das ações ofertadas e satisfação dos usuários. Mostrou, ainda em um ciclo intermediário, com 29.778 equipes avaliadas, uma oferta suficiente de ações materno-infantis, de prevenção do câncer feminino, de algumas condições crônicas como diabetes mellitus, porém, somente $55 \%$ das equipes atendendo usuários com transtornos mentais e $40 \%$ atendendo usuários com DPOC/Asma. Em 70\% das 24.055 UBS avaliadas, não estavam disponíveis os testes rápidos para gravidez, sífilis e HIV. E em $45 \%$ delas não estavam disponíveis a penicilina $G$ benzatina. Os parâmetros disponíveis nos diferentes módulos e ciclos de avaliação permitiram análises específicas, como é o caso da qualidade do prénatal de 6.125 mulheres, com resultados positivos como a utilização de sulfato ferroso em $96,5 \%$ das gestantes; a vacina antitetânica em 97\%, a solicitação de HIV em 94,0\%, menor para VDRL $(84,3 \%)$ e glicemia (83\%). Em relação às orientações e aconselhamentos como para aleitamento materno exclusivo (91\%), alimentação e peso (89\%) e cuidados com o bebê (86\%), bem como altos percentuais de pressão arterial $(98,7 \%)$ e altura uterina $(97,4 \%)$ aferidas, e a persistência de lacunas em relação ao exame de mamas (56,3\%), da cavidade oral (45\%) e de exame ginecológico $(44 \%)^{16}$. Ainda que possa parecer baixo o percentual de gestantes com exame da cavidade oral, esse é um achado correlacionado com a ampliação do acesso à saúde bucal, por meio da Saúde Bucal na APS, uma especificidade do modelo público brasileiro.

Muitas análises foram realizadas após a implantação do Programa Mais Médicos em 2013, principalmente em seu eixo de provisão a populações desassistidas, cuja abrangência foi marcante, em todas as capitais, nos 34 Distritos Sanitários Especiais Indígenas (DSEI) e em $82 \%$ dos municípios com proporção maior de pessoas em extrema pobreza ${ }^{17}$. Porém, seu caráter provisório, remuneração dos médicos por bolsa, fragmentação da rede de serviços e baixa capacidade da APS em coordenar o cuidado dos usuários na rede de serviços, mantém, ainda hoje, passados seis anos, alta rotatividade profissional e dificuldades em colocar a APS como prioridade nas gestões municipais.

O Brasil assume novos desafios, de acordo com suas mudanças demográficas e epidemiológicas, com a Agenda 2030 para o Desenvolvimento Sustentável e seus 17 Objetivos do Desenvolvimento Sustentável, de acordo com as diretrizes da Declaração de Astana, do ano de 2018, "Saúde em Todas as Políticas" 18 . Em relação ao Objetivo 3, de Saúde e Bem Estar, que trata tanto do controle dos fatores de risco e das doenças crônicas não transmissíveis, o Brasil, com exceção da contenção da obesidade, tem 


\section{APS em Revista}

Vol. 1, n. 3, p. 154/161 | Setembro/Dezembro - 2019

ISSN 2596-3317-DOI 10.14295/aps.v1i3.48

Mendonça, C. S.

alcançado todas às demais metas, como a redução da mortalidade prematura (30 a 69 anos) por DCNT em $2 \%$ ao ano; a redução da prevalência do tabagismo em $30 \%$, o aumento da cobertura de mamografia acima de $70 \%$ e do preventivo de colo uterino acima de $85 \%$ em mulheres das respectivas faixas etárias de rastreio; aumento da prática da atividade física no tempo livre em $10 \%$; aumento do consumo de frutas e hortaliças em $10 \%$ e redução do consumo abusivo de bebidas alcoólicas em $10 \%{ }^{19}$

As publicações científicas sobre APS no Brasil mudaram significativamente com o crescimento da ESF, tendo as publicações indexadas com os termos APS/atenção básica no Brasil crescido a partir de 2006 - ano da PNAB, e o número acumulado coloca o Brasil em um nível intermediário de publicações na área, quando comparado a países com sistemas de saúde orientados à APS ${ }^{20}$. Tal crescimento permitiu a realização de revisões sistemáticas em busca do impacto da Estratégia Saúde da Família, como é o caso da realizada por Bastos et al, de 1994 a 2016, cujo resultado demonstrou impacto na redução da mortalidade infantil e pós neonatal, na redução de internações sensíveis e, com menor rigor metodológico, alguns outros resultados como aumento da detecção de casos de hanseníase, de desnutrição infantil e aumento na vacinação antitetânica ${ }^{21}$.

Estudos publicados posteriormente, mostram efeito da SF na redução de internações sensíveis em crianças, na Bahia ${ }^{22}$ e a expansão da SF também esteve associada à redução de iniquidades de raça/cor na mortalidade no Brasil ${ }^{23}$.

A Figura abaixo representa a evolução da Saúde da Família no país, as principais agendas internacionais às quais o Brasil se comprometeu e que coincidiram com a evolução dessa política inclusiva, equânime e abrangente, e as políticas intersetoriais, a partir da Saúde da Família - na educação, ciência, tecnologia e pesquisa.

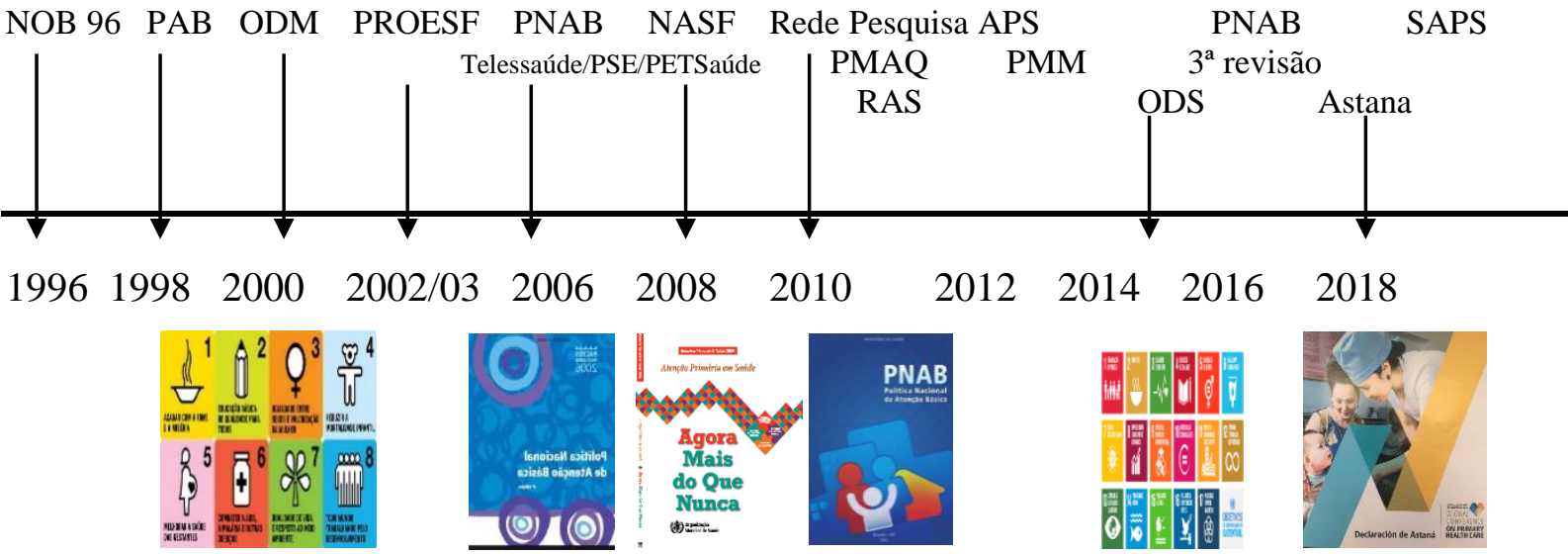

A continuidade dos avanços da Saúde da Família como política nacional está em debate nesse momento no país. Há a proposta de mudança no modelo de financiamento, somando os Pisos de Atenção Básica, fixo e variável, e transformando-os em uma capitação ponderada e pagamento por desempenho, em um cenário de subfinanciamento do SUS e de limitações de investimentos em políticas sociais, marcam o desafio das próximas décadas, para que os gestores municipais possam, enfim, colocar a APS/Saúde da Família como centro das redes de atenção. 


\section{APS em Revista}

Vol. 1, n. 3, p. 154/161 | Setembro/Dezembro - 2019

ISSN 2596-3317 - DOI 10.14295/aps.v1i3.48

Mendonça, C. S.

\section{REFERÊNCIAS BIBLIOGRÁFICAS}

(1) Brasil. Ministério da Saúde. Avaliação normativa do Programa Saúde da Família no Brasil: monitoramento da implantação e funcionamento das equipes de saúde da família: 2001-2002 / Secretaria de Atenção à Saúde, Departamento de Atenção Básica. - Brasília: Ministério da Saúde, 2004. 140 p. Série C. Projetos, Programas e Relatórios.ISBN 85-334-0770-X.

(2) BRASIL.Compromisso para Acelerar a Redução das Desigualdades na Região Nordeste e Amazônia Legal: Plano de Ações. Brasília, DF, 2009.

(3) Macinko J, Guanais FC, de Fatima M, de Souza $M$. Evaluation of the impact of the Family Health Program on infant mortality in Brazil, 1990-2002. J EpidemiolCommunity Health. 2006;60(1):13-9. pmid:16361449

(4) BRASIL. Ministério da Saúde. Banco Internacional para a Reconstrução e o Desenvolvimento - BIRD. Contrato de Empréstimo Externo no 7545-BR. Projeto de Expansão e Consolidação da Saúde da Família - PROESF. Fase 1, entre 2002 e 2007. Fase 2 entre 2009 e 2013. (http://bvsms.saude.gov.br/bvs/publicacoes/PROE SF.pdf)

(5) Conselho Nacional de Desenvolvimento Científico e Tecnológico, Ministério da Ciência e Tecnologia/ Ministério da Saúde. Edital MCTCNPq/MS-DAB/ SAS - no. 49/2005.

(6) HARTZ, Zulmira Maria de Araújo. Princípios e padrões em metaavaliação: diretrizes para os programas de saúde. Ciênc. saúde coletiva, Rio de Janeiro, v. 11, n. 3, p. 733-738, set. 2006

(7) FIGUEIRO, Ana Cláudia et al .Meta-evaluation of baseline studies of the Brazilian Family Health Strategy Expansion Project: a participatory and formative approach. Cad. Saúde Pública, Rio de Janeiro, v. 26, n. 12, p. 2270-2278, Dec. 2010.

(8) Barbosa ACQ (Coord). Saúde da Família no Brasil: Situação Atual e Perspectivas - Estudo Amostral 2008 - avaliação normativa do programa saúde da família no Brasil - monitoramento da implantação das equipes de saúde da família e saúde bucal. Belo Horizonte: Observatório de Recursos Humanos em Saúde Faculdade de
Ciências Econômicas da UFMG, 2009 (relatório final).

(9) BRASIL. Ministério da Saúde. Portaria GM 2226, que Institui, no âmbito da Política Nacional de Atenção Básica, o Plano Nacional de Implantação de Unidades Básicas de Saúde para Equipes de Saúde da Família.

(10) Giovanella L et al. A provisão emergencial de médicos pelo Programa Mais Médicos e a qualidade da estrutura das unidades básicas de saúde. Ciência \& Saúde Coletiva, 21(9):2697-2708, 2016.

(11) Projeto AQUARES - Avaliação de Serviços de Saúde no Brasil: Acesso e Qualidade da Atenção (2010). Universidade Federal de Pelotas UFPEL Acessado em 2019 nov 25. Disponível em: https://dms.ufpel.edu.br/aquares/wpcontent/uploads/2013/01/AquaresFINAL-.pdf

(12) White KL, Williams TF, Greenberg BG. The ecology of medical care. 1961. Bull N Y Acad Med 1996; 73(1):187-205.

(13) Green LA. The ecology of medical care.N Engl J Med, Vol. 344, No. $26 \cdot$ June 28, 2001.

(14) MALTA, DC et al. A Cobertura da Estratégia de Saúde da Família (ESF) no Brasil, segundo a Pesquisa Nacional de Saúde, 2013. Ciênc. saúde coletiva [online]. 2016, vol.21, n.2, pp.327-338.

(15) BRASIL. Ministério da Saúde. Portaria $\mathrm{n}^{\circ}$ 1.654, de 19 de julho de 2011. Institui, no âmbito do Sistema Único de Saúde, o Programa Nacional de Melhoria do Acesso e da Qualidade da Atenção Básica (PMAQ-AB) e o Incentivo Financeiro do $P M A Q-A B$, denominado Componente de Qualidade do Piso de Atenção Básica Variável - PAB Variável.

(16) Tomasi E, Fernandes PAA, Siqueira FCV et al. Qualidade da atenção pré-natal na rede básica de saúde do Brasil: indicadores e desigualdades sociais. Cadernos de saúde pública. 2017; 3(33) e00195815.

(17) Campos GWS e Junior NP. A Atenção Primária e o Programa Mais Médicos do Sistema Único de Saúde: conquistas e limites. Ciência\&saúdeColetiva. Vol 21 no 9, set 2016.

(18)Primary health care: transforming vision into action OPERATIONAL FRAMEWORK. Technical 
Vol. 1, n. 3, p. 154/161 | Setembro/Dezembro - 2019

ISSN 2596-3317-DOI 10.14295/aps.v1i3.48

Mendonça, C. S.

Series on Primary Health care. Acessado em: 2019

nov $25 . \quad$ Disponível em:

https://www.who.int/docs/default-

source/primary-health-care-

conference/operational-

framework.pdf?sfvrsn=6e73ae2a 2

(19) Fátima Marinho. $\mathrm{V}$ Fórum para o monitoramento do plano de ações estratégicas para o enfretamento das doenças crônicas não transmissíveis. Acessado em: 2019 nov 25. Disponível em: http://portalarquivos2.saude.gov.br/images/pdf/2 017/maio/16/5.Situacao-Epidemiologica-dasDCNT-no-Brasil.pdf

(20) Macinko, James and Mendonça, ClaunaraSchilling Estratégia Saúde da Família, um forte modelo de Atenção Primária à Saúde que traz resultados. Saúde debate, Set 2018, vol.42, no.spe1, p.18-37. ISSN 0103-1104

(21) Bastos ML; Menzies D; Hone T; Dehghani K and TrajmanA.,Impact of the Family Health Strategy on primary care sensitive health outcomes. PLOS ONE | August 7, 20171 / 14. https://doi.org/10.1371/journal.pone.0182336

(22) Pinto Junior EP; Aquino R; Medina MG; Silva MGCD. Efeito da Estratégia Saúde da Família nas internações por condições sensíveis à atenção primária em menores de um ano na Bahia, Brasil. Cad Saude Publica;34(2):e00133816, 2018 Feb 19.

(23) Hone T; Rasella D; Barreto ML; Majeed A; Millett C. Association between expansion of primary healthcare and racial inequalities in mortality amenable to primary care in Brazil: A national longitudinal analysis. PLoS Med;14(5):e1002306, 2017 


\section{ABSTRACT}

Background: Synthesize the evaluation processes carried out by the Ministry of Health throughout the expansion of Family Health and the commitments assumed by Brazil as part of the global efforts to ensure universal access to health services

\section{Methods: Opinion article}

Results: The results indicate that the increased access of the Brazilian population to the ESF, a model of primary health care that takes into account the social and structural determinants of health, were decisive in increasing access, equity and impacted on the classic indicators of maternal and child health and in reducing early deaths from chronic non-communicable diseases.

Conclusions: The achievement of a minimum infrastructure, sufficient inputs and professionals, puts our country on a new level of global leadership, which is to place APS/Family Health as the center of care networks across the country. Giving visibility to what has already been achieved is one of the ways to ensure the sustainability of the ESF and the SUS, in a restrictive context of social policies and economic austerity, which we are facing at the beginning of the 21st century

Keyword: Primary Health Care; Unified Health System; Family Health; access to health services 